\title{
Politique
}

\section{L'argumentation stratégique dans la communication politique : le débat télévisé L’Allier - Bertrand}

\section{Gilles Gauthier}

Numéro 17, hiver 1990

Les nouveaux enjeux du politique

URI : https://id.erudit.org/iderudit/040650ar

DOI : https://doi.org/10.7202/040650ar

Aller au sommaire du numéro

Éditeur(s)

Société québécoise de science politique

ISSN

0711-608X (imprimé)

1918-6584 (numérique)

Découvrir la revue

Citer cet article

Gauthier, G. (1990). L'argumentation stratégique dans la communication politique : le débat télévisé L’Allier - Bertrand. Politique, (17), 113-141.

https://doi.org/10.7202/040650ar d'utilisation que vous pouvez consulter en ligne.

https://apropos.erudit.org/fr/usagers/politique-dutilisation/ 


\title{
L'argumentation stratégique dans la communication politique: le débat télévisé L'Allier - Bertrand
}

\author{
Gilles Gauthier \\ Université Laval
}

Toutes les billevesées de la métaphysique ne valent pas un argument adhominem.

Diderot

Depuis l'affrontement Kennedy - Nixon, lors des élections présidentielles américaines de 1960, le débat médiatique entre chefs de partis est devenu l'un des événements clés du déroulement des campagnes électorales dans les démocraties occidentales. Il a, de ce fait, donné lieu à un assez grand nombre d'études et de critiques centrées sur ses effets ou conséquences, tels que son impact sur le résultat d'un scrutin ou son incidence sur la vie démocratique. Jusqu'à maintenant cependant, la structuration même du débat électoral n'a fait l'objet que de quelques analyses.

C'est à ce dernier aspect qu'est consacré le présent texte. J'y poursuivrai deux objectifs: la caractérisation d'un élément essentiel du débat, que j'appelle «l'argumentation stratégique», et le compte 
rendu de son application dans un cas exemplaire, celui du débat télévisé ayant opposé MM. Jean-Paul L'Allier et Jean-François Bertrand, candidats à la mairie lors des élections municipales tenues à Québec en novembre 1989. Cette recherche à double volet prend appui sur un arrière-plan théorique, soit la communication politique, et sur l'hypothèse selon laquelle le débat électoral serait de nature «communicationnelle».

\section{La communication politique}

La politique, au moins dans quelques-unes de ses activités les plus éclatantes, est aujourd'hui de plus en plus considérée comme une activité de communication publique. Cette vue des choses ne se réduit pas au simple constat de l'importance prise par les techniques de communication dans la vie publique contemporaine. Plus fondamentalement, elle implique une reconnaissance de la nature «communicationnelle» de l'action politique, conçue essentiellement comme une opération discursive de relations sociales à finalité persuasive.

Peut-être la politique a-t-elle toujours été d'ordre «communicationnel», mais ce caractère est plus net à l'ère actuelle des communications de masse et de la culture de communication. Cette perception conduit même déjà à une dénonciation: d'aucuns s'insurgent contre ce qu'on peut appeler la «communicationalisation», à leurs yeux trop grande, de la pratique politique contemporaine ${ }^{1}$.

Le débat électoral est certainement l'une des formes du discours politique dont la nature «communicationnelle» apparaît le plus clairement. Cette confrontation, le plus souvent entre chefs de partis, consiste en un exercice de relations multiples, dont l'ob-

1. Je ne donnerai ici qu'un seul exemple: dans L'Actualité d'avril 1989, André Noël signe un article intitulé «La démocratie peutelle survivre à la télévision?», dans lequel il vitupère l'utilisation abusive des médias, en particulier la télévision, lors de la dernière campagne électorale fédérale de 1988. 
jectif ultime est de convaincre un électorat d'accorder son appui à l'un ou l'autre candidat en lice. Le débat électoral constitue en fait la version moderne de l'assemblée contradictoire d'antan: il s'agit d'un «duel» au cours duquel les opposants cherchent tout autant à faire prévaloir leur point de vue qu'à discréditer celui de l'adversaire.

\section{Débat électoral et communication}

À plus d'un titre, le débat est une opération de communication. D'abord, bien sûr, il est en lui-même une production médiatique, puisqu'il prend toujours la forme d'une émission de télévision ou de radio. Mais au-delà de la forme matérielle, un débat représente un sujet multiforme d'actualité électorale. Il donne lieu à des comptes rendus journalistiques détaillés et à de savantes analyses éditoriales, aussi bien dans la presse écrite que dans la presse électronique. Même les à-côtés d'un débat deviennent matière à information: la concurrence entre les diffuseurs, les réactions et exigences des chefs de partis, les négociations et le marchandage qui s'ensuivent, la préparation des participants et jusqu'à leur arrivée en studio font l'objet de nouvelles, souvent à la «une» ou en manchette, et de commentaires. De même, le résultat, c'est-à-dire la détermination d'un vainqueur, et les conséquences éventuelles du débat donnent lieu à une évaluation journalistique. L'électorat luimême est appelé à se prononcer sur l'issue du débat à des tribunes téléphoniques ou dans des sondages scientifiques dont les données deviennent ensuite objets de nouvelles. L'importance médiatique du débat électoral est telle que même s'il n'a pas lieu, comme lors des dernières élections québécoises de septembre 1989, il n'en génère pas moins une quantité importante de nouvelles: supputations chez les journalistes; dénonciations et justifications chez les hommes politiques.

L'hypothèse de base dans le présent texte est que l'analyse de la nature «communicationnelle» d'un débat électoral peut se faire suivant trois points de vue distincts: la présentation et l'exploitation d'une certaine image des hommes politiques; l'exposition et 
la défense de leurs idées, que j'appelle l'«argumentation idéelle»; et un type distinct d'argumentation auquel le nom d'«argumentation stratégique» me semble convenir. La recherche menée ici porte exclusivement sur ce dernier aspect. Ne serait-ce que pour marquer plus nettement ce qui le distingue, il convient toutefois de parler brièvement de l'image et de «l'argumentation idéelle», auxquelles il s'oppose.

Un débat électoral peut d'abord être considéré comme une entreprise de séduction. On met d'ailleurs souvent en évidence l'importance de l'image projetée par un participant en s'attardant à sa présentation physique. Certains prétendent, par exemple, que son teint hâlé a favorisé Kennedy contre Nixon en 1960; que son assurance a fait marquer à Lesage des points contre Johnson en 1962, que la bonhomie de Reagan a joué contre Mondale en 1984, que l'attitude agressive de Mulroney a nui à Turner en 1984; et que la fermeté de Turner s'est retournée contre Mulroney en 1988. Jusqu'à maintenant, l'examen de cette sorte d'impact en est encore au stade des généralisations intuitives. Il faudrait développer une typologie des différents aspects constitutifs d'une image.

Dans un débat, les participants cherchent également à convaincre l'électorat de la justesse de leur point de vue d'ensemble sur la collectivité ou la société concernée. Ils portent un diagnostic sur les problèmes qu'affronte cette société et, pour les résoudre, ils défendent une idéologie politique en mettant de l'avant un programme d'action. Même si on semble le souligner de moins en moins, un débat porte sur un certain «contenu». Il consiste, au moins partiellement, en un échange d'«idées» où sont discutés des choix de société.

La propagation des idées est toutefois relative à leur expression: une idée bien exposée et bien défendue va rallier plus d'adhérents qu'une idée supérieure qui serait mal présentée et moins bien défendue. Dans sa mise en forme et son exposition, la pensée politique donne ainsi lieu à une argumentation d'idées ou «argumentation idéelle». L'analyse proposée ici ne s'attache pas à ce type d'argumentation sans pour autant présupposer ou laisser entendre que la confrontation d'idées est exclue de tout débat. 
Dans la suite de cet article, on essaiera de caractériser le plus précisément possible le troisième aspect qui fait du débat une opération de communication publique, c'est-à-dire «l'argumentation stratégique». On testera également la définition que j'en propose en procédant à un examen du débat l'Allier - Bertrand. Cette «analyse de cas» veut mettre en lumière, entre autres choses, les raisons pour lesquelles Jean-Paul L'Allier est sorti vainqueur de son affrontement avec son adversaire Jean-François Bertrand.

\section{L'argumentation stratégique: proposition de définition}

Le cadre théorique sur lequel s'appuie l'analyse qui suit est très large puisqu'il s'agit de «la théorie contemporaine de l'argumentation». On peut définir celle-ci, grosso modo, comme l'étude des moyens discursifs employés pour convaincre ou persuader un auditoire. De façon normative, l'un des principaux objectifs de la théorie de l'argumentation est d'analyser les diverses modalités d'évaluation des arguments. L'argumentation est devenue, au cours des dernières décennies, un champ d'étude important dans toutes les disciplines qui de près ou de loin s'intéressent au langage, au discours et, de façon plus abstraite, à la pensée. L'une des prémisses de la théorie de l'argumentation est que l'art d'argumenter ne relève pas seulement de la raison ou, à tout le moins, de la conception rationaliste de la raison. Autrement dit, un argument n'est pas une démonstration d'évidence ou une déduction de nécessité, calquée sur le calcul mathématique. Pour appuyer cette affirmation, on invoque souvent le fait qu'une inférence logiquement valide ne suffit pas toujours à convaincre un auditoire, lequel se laissera parfois persuader plutôt par une considération qui manque de cohérence formelle.

La théorie contemporaine de l'argumentation semble se proposer d'élargir la notion de raison et de décloisonner la preuve destinée à convaincre. (C'est ainsi qu'elle s'apparente globalement à l'approche pragmatique). Elle s'oppose au rationalisme et au logicisme qui, sous diverses formes, ont présidé pour une bonne 
part au développement du mode de pensée occidental en bornant les ressources de l'intellect à la seule raison logique, démonstrative ou mathématique.

À la théorie contemporaine de l'argumentation se rattache un grand nombre de recherches plutôt disparates. Certains de ces travaux conviennent particulièrement à l'étude du discours politique considéré comme pratique de communication. Ils peuvent être regroupés en deux sous-théories: la «théorie de l'argumentation communicationnelle» et la «théorie de l'argumentation fallacieuse».

Des chercheurs, qui se donnent pour tâche d'examiner l'usage courant des arguments, étendent leur champ d'intérêt aux médias de masse, aux professions de communication sociale et autres modes d'expression publique. Vernon Jensen (1981), Michael Sproule (1980) et Howard Kahane (1988) ont ainsi développé un nouveau champ d'application de la théorie de l'argumentation, soit la communication publique. Ils analysent, par exemple, le fonctionnement typique de l'argumentation en publicité, en journalisme (dans les textes de nouvelles, les textes d'analyse et les éditoriaux), dans différents types de prose publique (les essais et divers textbooks) et même dans les textes scientifiques, la littérature, le cinéma, la chanson et autres formes d'expression. Le discours politique n'échappe évidemment pas à cette investigation: Sproule consacre quelques pages à l'analyse de l'allocution politique; Kahane examine quelques traits du débat politique et, plus spécifiquement, du débat parlementaire.

Un second objet de recherche a trait aux formes d'argumentation fallacieuse, aux sophismes ou encore au pseudo-raisonnement. Il a donné lieu à ce qu'on appelle en anglais la théorie des fallacies. Il faut dire, d'abord, que l'examen des procédés fallacieux d'argumentation ne date pas d'hier. Aristote en avait dressé un inventaire et, afin de confondre les sophistes, avait mis au jour le caractère illogique ou illusoire de quelques-uns de leurs modes d'argumentation. L'analyse des fallacies a été reprise de façon spectaculaire à notre époque par Charles Hamblin (1970) et poursuivie dans différentes directions principalement par Ralph Jonson et Anthony Blair (1977), Morris Engel (1980), Douglas Walton et John 
Woods (1982), Vernon Jensen (1981), Douglas Walton (1987) et Howard Kahane (1988). Un grand nombre de travaux sur l'argumentation fallacieuse prennent expressément pour objet les discours politiques. Par exemple, Walton et Woods (1982), pour qui le modèle idéal d'argumentation est le débat (the Debate), compris comme un échange structuré et organisé suivant certaines règles et procédures, examinent l'utilisation d'arguments ad populum (appels aux sentiments et aux préjugés d'un groupe) lors de débats parlementaires. Certains autres échanges parlementaires donnent aussi lieu à des cases studies: Walton (1987), pour ne donner qu'un seul exemple, traite du recours aux fallacies ad hominem (l'attaque contre la personne), ad verecundiam (l'appel à l'autorité) et ignoratio elenchi (une réplique non pertinente) dans quelques débats à la Chambre des communes du Canada.

Il existe au moins une étude centrée sur l'argumentation fallacieuse en débat électoral. L'auteur, Gary Jason (1988), procède à une analyse comparée des débats présidentiels ayant opposé dans un cas Kennedy et Nixon en 1960 et, dans l'autre, Reagan et Mondale en 1984. Il met alors en évidence quatre procédés fallacieux: ignoring the Issue (omission de certains enjeux en évitant le débat sur certaines questions et en se cantonnant dans des généralités abstraites); false cause (imputation à l'opposant de la responsabilité de tout ce qui a pu survenir pendant l'exercice de son mandat); ad populum (attitude populiste ou appel indu au sens patriotique de l'électorat); ad hominen attacks (prise à parti de l'adversaire lui-même).

L'argument ad hominem est généralement considéré comme l'un des principaux types de fallacies. Grosso modo, il consiste à mettre en cause la personne d'un locuteur plutôt que ses idées. Comme tel, il fut formellement présenté et précisé par Locke dans son Essay Concerning Human Understanding. Depuis, il fait l'objet d'une attention soutenue en théorie des fallacies, et d'un intérêt moins marqué en théorie logique où sa nature et sa portée donnent lieu à des débats plus qu'à des consensus. Certains n'admettent pas qu'une attaque ad hominem soit un véritable argument, au moins au sens strict du terme. En fait, on propose différentes définitions de l'argument ad hominem. Pour Locke, cet 
argument est la mise en évidence de la contradiction formelle qu'il y a pour un locuteur à soutenir telle idée compte tenu de l'ensemble de sa position préalable. L'objectif visé est de discréditer l'idée du locuteur en s'en prenant expressément à sa personne. Voici la formulation, devenue classique, que donnent Stephen Toulmin, Richard Riecke et Allan Janik (1984) de cette conception de l'argument ad hominem:

The argument against the person is the fallacy of rejecting the claims a person advances simply on the basis of derogatory facts (real or alleged) about the person making the claim. Such a procedure takes for granted that the substance or content of a claim is essentially connected with the character or situation of the claimant (144).

C'est cette définition informelle de l'argument ad hominem que retient la théorie des fallacies.

Le discours politique est manifestement l'un des lieux de prédilection de l'argumentation ad hominem, «l'homme», comme on dit, étant en politique aussi important que «les idées». Aussi n'est-il pas étonnant qu'un grand nombre de travaux en théorie des fallacies cherchent à caractériser l'argument ad hominem par rapport au discours politique. Walton (1987), par exemple, étudie ce qu'il appelle le theorical context of Ad Hominem Attacks relativement, entre autres choses, à l'allocution politique et au débat parlementaire. De même, comme nous l'avons déjà vu, Jason (1988) examine le recours à l'attaque ad hominem dans certains débats électoraux.

Les diverses orientations de recherche qui marquent la théorie contemporaine de l'argumentation donnent évidemment lieu à des définitions et à des caractérisations fort différentes des notions d'argumentation et d'argument ${ }^{2}$. La définition qui pourrait bien être

2. Il n'est même pas sûr, en vérité, que tous les travaux qui se réclament de l'étude de l'argumentation portent véritablement sur le même objet. La très grande extension donnée au concept d'argument pose même un problème épistémologique au développement de la théorie de l'argumentation: à vouloir embrasser un champ d'analyse 
à la fois la plus riche et la plus opérationnelle est proposée par Frans van Eemeren, Rob Grootendorst et Tjark Kruiger (1987):

Argumentation is a social, intellectual, verbal activity serving to justify or refute an opinion, consisting of a constellation of statements and directed towards obtaining the approbation of an audience (p. 7).

Il semble que cette définition prenne en compte tous les éléments essentiels de l'argumentation et les agence de façon précise et succincte. Tout en conservant la substance, je propose de la paraphraser ainsi:

L'argumentation consiste en un ensemble organisé d'énoncés - exprimant par le langage une série de propositions dans un certain contexte d'énonciation - auquel un argumentateur a recours afin de justifier ou d'infirmer une opinion dans le but de gagner l'adhésion d'un auditoire.

Si cette caractérisation de l'argumentation ne semble pas d'une grande utilité pour l'analyse de l'image dans un débat électoral (pour la simple et bonne raison que l'exploitation d'une image ne donne pas lieu à une argumentation, au moins au sens strict du terme), elle pourrait par contre s'appliquer à l'examen de l'argumentation idéelle, support de la pensée politique mise de l'avant par un argumentateur. Pour analyser le troisième aspect permettant de situer les débats électoraux dans le champ de la communication, aspect que j'appelle l'«argumentation stratégique», il faut raffiner quelque peu la définition d'Eemeren Grootendorst et Kruiger.

Un débat n'est ni un pur échange d'idées ni un cadre «neutre» ni une enveloppe «sans épaisseur» servant uniquement à mettre en évidence un certain contenu. Un débat, c'est aussi un type particulier de rapport ou d'interrelation: véritable affrontement, confrontation, ou lutte entre des participants qui deviennent par le fait même des opposants. La nature conflictuelle du débat engendre un type d'argumentation différent de l'argumentation idéelle. Pour

trop disparate et trop multiforme, elle risque de voir se diluer son soidisant objet d'étude. 
se gagner la faveur d'un électorat, les protagonistes cherchent non seulement à faire valoir la supériorité de leurs idées sur celles de l'adversaire, mais aussi, sur un plan formel, à sortir vainqueurs de l'affrontement. Or, la défense d'idées et l'exposition d'une certaine image, si elles peuvent contribuer à ce résultat, n'en sont pas les éléments déterminants. L'issue dépend, dans un grand nombre de cas, de l'utilisation efficace de certains procédés qui ont spécifiquement trait au cadre oppositionnel du débat. Par exemple, un argumentateur pourrait essayer de mettre en cause le leadership de son adversaire en insistant sur les critiques dont il fait l'objet à l'intérieur même de son parti politique.

C'est l'ensemble de ces procédés que je propose de grouper sous l'appellation d'«argumentation stratégique». L'adjectif doit être entendu dans un sens technique dérivé de son sens lexical. Un débat électoral représente une forme d'affrontement similaire à la guerre. Aussi apparaît-il justifié de parler de «stratégie» à son sujet dans la mesure où le terme est utilisé dans une acception élargie. L'argumentation idéelle et l'exploitation d'une image dépendent également de considérations appelées «stratégiques» quand on veut suggérer que leur recours et leur mode d'exposition relèvent d'un plan d'ensemble et visent à l'atteinte d'objectifs bien identifiés. Cette dimension «stratégique» differe totalement de l'argumentation stratégique; celle-ci se rattache substantiellement à la nature conflictuelle du débat. L'argumentation stratégique, c'est ce qui, dans un débat, relève de l'éristique, c'est-à-dire de la controverse en tant que telle, sans véritable référence à un contenu politique. Elle a trait au positionnement purement formel que les argumentateurs prennent ou veulent prendre l'un vis-à-vis de l'autre dans leur rapport de forces.

Au sens qui lui est donné ici, l'argumentation stratégique se distingue des concepts d'«analyse stratégique» ou de «planification stratégique» propres aux sciences politiques et administratives. Elle n'est pas reliée à la recherche d'une certaine forme de rationalité et n'a donc pas une portée macro-analytique. Plus modestement, la notion d' «argumentation stratégique» dénote les procédés discursifs qui cherchent à emporter l'adhésion par une attaque for- 
melle de l'adversaire (ou par une défense contre une semblable attaque de la part de l'adversaire).

Intuitivement, on peut dire que les procédés d'argumentation ayant spécifiquement trait au cadre oppositionnel du débat sont relatifs soit à une question de crédibilité, soit à une certaine exaltation émotive. L'argumentation stratégique semble également pouvoir être utilisée dans trois sens: l'apologie, l'attaque et la défense. Un argumentateur peut, en recourant à quelque trait de crédibilité ou d'émotion, soit mettre en valeur sa propre candidature, soit mettre en cause celle de l'opposant, ou encore rétorquer à une attaque semblable de l'adversaire.

Afin d'illustrer cette caractérisation globale de l'argumentation stratégique, voici un premier exemple précis et réel. À un certain moment du débat radiophonique, l'opposant de René Lévesque en 1976, Robert Bourassa, a cherché à contester la compétence administrative du chef du Parti québécois et de quelques autres de ses principaux dirigeants en invoquant la disparition prématurée et récente du quotidien Le Jour qu'ils dirigeaient. L'objectif de Bourassa était clair: saper la crédibilité, en matière administrative et plus largement financière, de Lévesque et de certaines autres têtes d'affiche du PQ (nommément Jacques Parizeau et Yves Michaud). Il s'agissait là d'un procédé éristique qui n'avait, bien sûr, rien à voir avec les divergences politiques opposant Bourassa et Lévesque. Celui-ci, visiblement bien préparé à faire face à une telle attaque, ne resta sur la défensive que le temps de préciser que l'aventure du quotidien souverainiste n'avait toujours été qu'une entreprise d'ordre privé pour aussitôt contre-attaquer et mettre en doute à son tour la compétence administrative de Bourassa en lui reprochant la faillite financière du club La Réforme. Désarçonné par cette vive riposte à laquelle il ne s'attendait visiblement pas, Bourassa non seulement dut battre en retraite dans son argumentation, mais parut manifestement ébranlé et perdit certainement des points. Cet exemple montre que l'utilisation d'un argument stratégique comporte un certain risque et peut entraîner un effet boomerang.

Compte tenu des précisions apportées ci-dessus à propos de l'argumentation stratégique et à partir de la définition générale de 
l'argumentation que donnent Eemeren, Grootendorst et Kruiger, on peut proposer une nouvelle définition:

L'argumentation stratégique consiste en un ensemble organisé d'énoncés - exprimant par le langage une série de propositions dans un certain contexte d'énonciation - auquel un argumentateur a recours afin de conforter sa crédibilité (ou de saper celle de son adversaire) ou de susciter l'émotion d'un auditoire dans le but de gagner son adhésion.

\section{Le débat L'Allier - Bertrand}

Afin de vérifier l'exactitude de la définition proposée, je l'appliquerai maintenant au débat qui a opposé Jean-Paul L'Allier et Jean-François Bertrand, les deux candidats à la mairie de Québec lors des élections municipales du 5 novembre 1989. Quelles formes précises l'argumentation stratégique a-t-elle revêtues lors de ce débat particulier?

Cet affrontement électoral a mis aux prises deux formations politiques dirigées chacune par un nouveau chef. Après douze ans d'exercice du pouvoir, Jean Pelletier quittait, en 1989, le Progrès civique. Il y fut remplacé par Jean-François Bertrand, qui n'était pas un militant du parti mais qui, selon les sondages, bénéficiait d'une très grande popularité. De son côté, le Rassemblement populaire, en se donnant pour chef Jean-Paul L'Allier, cessait d'être seulement un parti idéologique et se donnait un moyen d'accéder au pouvoir.

On a dit de la lutte électorale mettant aux prises L'Allier et Bertrand qu'elle a été une «bataille de communicateurs». Les deux aspirants à la succession du maire sortant avaient été ministres des Communications et tous deux s'étaient ensuite dirigés dans le domaine des communications, Bertrand à la radio, L'Allier à la direction d'un bureau-conseil. On pouvait donc s'attendre à ce que la campagne électorale mette l'accent sur la communication.

Ce fut le cas particulièrement lors du débat télévisé du 24 octobre. Ce «débat des chefs» fut présenté en direct de 21 heures à 
22 heures sur les ondes de CBVT, Radio-Canada - Québec. La formule retenue pour l'émission fut l'exposé traditionnel de l'un et l'autre candidats, suivi des questions posées par un auditoire en studio, et de l'épilogue des deux adversaires.

Faisant mentir l'idée reçue selon laquelle un exercice du genre n'a pas beaucoup d'incidence sur les résultats d'un scrutin, ce débat fut, de l'avis de tous les observateurs, un point tournant sinon l'élément crucial de cette course à la mairie de Québec ${ }^{3}$. Bertrand, qui jusque-là menait largement dans les sondages, allait se voir moins de deux semaines plus tard totalement écrasé, L'Allier obtenant près de $60 \%$ des voix et le Rassemblement populaire faisant élire 16 conseillers sur 21 .

Il est difficile et peut-être même impossible de déterminer avec précision ce qui a pu contribuer à un tel renversement. Faute d'éléments explicatifs mesurables et vérifiables, on peut dire de façon intuitive seulement que le débat fut la raison déterminante de la remontée de L'Allier. Il semble toutefois légitime de considérer qu'il en fut un déclencheur important. Son impact sur la fin de la campagne fut indéniable: à partir du débat, Bertrand perdit beaucoup de confiance et L'Allier se mit à afficher l'attitude sereine du vainqueur.

Le débat L'Allier - Bertrand fut donc un exercice de communication concluant. Mais sous quels aspects? Il ne semble pas, contrairement à des débats antérieurs que l'image des deux candidats y ait joué un grand rôle: sans être impeccable, leur image ne présentait pas de défauts notables ni surtout de contraste. Certainement différents l'un de l'autre à plusieurs égards, L'Allier et Bertrand sont par ailleurs du même type physique général. Le style d'homme public qu'ils incarnent est même assez semblable: ils sont tous deux posés dans leurs gestes et leurs propos, ni vraiment flamboyants ni spectaculaires, ni non plus d'allure populiste; ils ont tous deux une certaine pudeur ou réserve dans l'expression des émotions et ne répugnent pas à traiter, disons «intellectuelle-

3. Les deux quotidiens de Québec, Le Soleil et Le Journal de $Q u e ́ b e c$ déclarèrent L'Allier vainqueur du débat. L'Allier lui-même convint avoir largement profité de l'événement. 
ment», de certaines questions d'actualité. Les images de L'Allier et Bertrand présentent par ailleurs des différences notables. Le premier fait plus «bourgeois», plus «sérieux» et, à certains égards, inspire davantage confiance. Le second a plutôt l'air bon enfant, mais il semble peut-être moins crédible. Malgré tout, leurs similitudes ont probablement atténué l'impact de leur image politique au cours du débat.

Leurs divergences d'idées a été d'une plus grande importance. Le débat a bien montré que L'Allier et.Bertrand ne partageaient pas le même avis sur des questions importantes: le développement urbain et plus spécifiquement le projet de la «Grande Place», la gestion et la fiscalité municipale de même que la pratique démocratique. Les deux candidats n'ont d'ailleurs pas cherché à cacher leur opposition, chacun s'efforçant au contraire de justifier sa position. À la lumière des résultats du scrutin, il semble que l'argumentation idéelle développée par L'Allier ait été supérieure à celle de Bertrand et qu'elle ait produit un plus grand impact. Je ne veux pas ici démêler les éléments qui ont pu compter à cet égard. L'argumentation stratégique est le seul point qui m'intéresse.

L'une des particularités qui fait du débat L'Allier - Bertrand un débat exemplaire est la précision de l'argumentation stratégique qu'ils ont l'un et l'autre fait valoir. En fait, chacun des deux argumentateurs a utilisé une seule argumentation stratégique, aux contours tout à fait nets. Comme nous le verrons, cette argumentation unitaire a, en quelque sorte, servi de support ou de pivot à leur argumentation idéelle. C'est le choc de ces deux argumentations qui a déterminé l'allure du débat et qui permet de formuler quelques hypothèses analytiques sur son résultat et son impact.

Il est clair que les argumentations respectives de L'Allier et de Bertrand appartiennent au même type général: celui que la théorie de l'argumentation fallacieuse nomme fallacie ad hominem. Les candidats ont tous deux cherché à ébranler la crédibilité de l'adversaire, Bertrand en recourant au procédé de mise en contradiction, L'Allier à celui de la culpabilité par association. 
Bertrand: l'attaque ad hominem par la mise en contradiction

Tout au long du débat, Bertrand accuse L'Allier de s'être contredit en changeant d'avis sur de nombreuses questions. Ainsi, dès le début de son exposé, Bertrand se lance dans une dénonciation de «... l'improvisation, l'incohérence et la contradiction...» manifestées par L'Allier et le Rassemblement populaire. Un leitmotiv marque les interventions de Bertrand: il reproche à L'Allier de toujours «...changer son fusil d'épaule ...». Il martelle l'accusation en alléguant que de tels revirements sapent la confiance de l'électorat:

En politique, tout est question de confiance.

Comment pouvons-nous faire confiance à un parti politique qui a changé son fusil d'épaule sur un ensemble de questions qui sont très importantes?

À quelques reprises, Bertrand énumère une série de sujets à propos desquels L'Allier aurait fait volte-face: l'incinérateur municipal, l'environnement, le développement, la fiscalité et la «Grande Place». C'est sur les deux dernières questions que Bertrand cherche à étayer son attaque avec le plus de précision.

Il évoque deux propositions différentes que L'Allier aurait prétendument formulées en remplacement du projet de la «Grande Place». Après avoir d'abord lancé l'idée d'ériger «... un centre culturel avec le regroupement des universités ...», L'Allier aurait, en fin de campagne, encouragé un second projet de développement encore plus imprécis. Au sujet de la fiscalité, Bertrand soutient que L'Allier et son parti «... ont changé leur fusil d'épaule trois fois ...»:

Un jour, on les gelait, les taxes; le lendemain, on les diminuait; et ce que l'on sait maintenant, c'est qu'ils vont les augmenter. Trois discours, en l'espace de trois jours!

Bertrand pousse l'attaque jusqu'à prétendre que L'Allier aurait voulu être chef du Progrès civique avant de briguer ce titre au Rassemblement populaire. Vers la fin du débat, en réaction à une 
offensive de L'Allier, qui l'associait à l'administration sortante du maire Pelletier, il posa à celui-ci la question suivante:

Comment se fait-il que vous ayez tenté, deux ans avant les élections, de devenir chef du Progrès civique?

L'attaque ad hominem dont use Bertrand est de la forme la plus classique, c'est-à-dire la plus simple et la moins subtile qui soit. De par sa caractérisation même, suivant tout autant la définition lockienne que la définition contemporaine, un argument $a d$ hominem consiste à mettre en évidence le manque de cohérence d'un locuteur afin de souligner sa faible crédibilité. C'est essentiellement et strictement ce à quoi s'emploie Bertrand: il essaie de discréditer la candidature de L'Allier en démontrant que celui-ci se contredit sur plusieurs sujets.

Une seconde observation s'impose. Fait surprenant, Bertrand n'a pas eu recours à cette forme d'argumentation ad hominem particulière dite de «l'homme de paille» (straw man). Selon la théorie des fallacies (Johnson et Blair (1983) et Walton (1987)), ce procédé consiste à déformer les vues d'un opposant en lui attribuant une position contestable ou discutable et par conséquent plus aisément critiquable. Kahane (1988) précise qu'un argument straw man ramène l'adversaire à une position similaire plus faible et donc plus facilement attaquable, ou contrefait carrément une vue adverse en l'associant à un symbole détesté. Kahane donne l'exemple de certains discours de Richard Nixon dans lesquels il insinue que ses opposants et leurs idées se rapprochent d'une idéologie radicale, le communisme par exemple.

L'histoire même du Rassemblement populaire et son propre «passage» au Progrès civique (un ancien ministre péquiste maintenant appuyé par les libéraux) auraient pu inciter Bertrand à évoquer, au sujet de son adversaire, le spectre du gauchisme. Il aurait de cette façon pu faire valoir que, même dirigé par un «modéré» ou un «réformiste», le «Ras pop» (aurait-il pu ironiquement spécifier) continuait d'être une formation qui faisait place aux militants idéalistes sinon «agitateurs» et aux idées trop «socialistes». Le plus loin qu'il est allé dans cette direction a été d'affirmer, de manière peu appuyée et exactement dans la foulée de sa mise en contra- 
diction, que le choix des électeurs devait se faire entre «... les administrateurs du Progrès civique et les improvisateurs du Rassemblement populaire». L'Allier semblait d'ailleurs s'attendre à une attaque de type straw man: il prend lui-même les devants avec l'intention évidente de «démarginaliser» ou de dédouaner sur sa gauche le Rassemblement populaire, par exemple en précisant «On n'est pas des granolas».

L'Allier réagit très vivement à la mise en contradiction que lui sert Bertrand. Il ne cherche pas à contrer vraiment cette argumentation stratégique, à la façon d'un judoka, il s'efforce plutôt de miner la crédibilité de son adversaire en utilisant l'élan qui met la sienne en cause. L'Allier ne se défend pas contre les «accusations» d'incohérence et d'improvisation que lui adresse Bertrand, mais il profite de l'ouverture qui lui est offerte pour le contre-attaquer. Il ne prend pas la peine d'expliquer et de justifier sa position dans les dossiers de la «Grande Place» et de la fiscalité. Il préfêre se rabattre à ce propos sur le programme du Rassemblement populaire «... qui est là depuis longtemps». L'Allier adopte une attitude plus offensive, pour ne pas dire agressive. Il somme Bertrand d'arrêter de "picosser» et lui reproche de rapporter ses propos de façon tronquée et de le citer hors contexte, bref de ne pas considérer la position d'ensemble du Rassemblement populaire:

Vous allez arrêter de me faire dire des choses que je n'ai pas dites. Vous allez écouter ce que je dis. Arrêtez de prendre des petites découpures ici et là et écoutez toutes les déclarations.

La réaction la plus cinglante de L'Allier est déclenchée quand Bertrand lui prête l'ambition de devenir chef du Progrès civique. Il rétorque tout de go: «Vous faites du fantasme sur les autres», et se lance dans une contre-offensive dont il ressort, noms et dates à l'appui, que c'est plutôt Bertrand qui a fait des avances au Rassemblement populaire. 
L'Allier: l'attaque ad hominem de culpabilité par association

L'Allier ne se contente pas de réagir à l'attaque de Bertrand. Pour discréditer à son tour la candidature de son opposant, il lance sa propre argumentation stratégique sur le thème: le changement vaut mieux que la continuité. Tout au long du débat, L'Allier propose cette alternative comme enjeu essentiel de l'élection en associant sa candidature à un renouveau. Dans son exposé de départ, il fait valoir la nécessité «... [d'un] changement, [d'un] vrai changement...»; son exhortation finale reprend le même accent: «Il faut changer pour vrai». «Changer pour vrai» était d'ailleurs le principal slogan électoral du Rassemblement populaire.

La thèse de la nécessité d'un changement véritable, L'Allier l'appuie sur une démonstration en deux temps. Il s'efforce d'abord de démontrer que le Progrès civique est incapable de renouvellement puis que Bertrand est associé au passé du parti et par le fait même à l'administration sortante. L'Allier développe donc lui aussi une argumentation stratégique de mise en accusation par association.

À la façon d'un constat plus que d'une dénonciation, L'Allier attire l'attention sur les inévitables effets de «... 25 ans de pouvoir d'un parti ...» qui «... devient, avec le temps un peu sourd ...» et «... se ferme à la démocratie...». En conclusion, il déclare que toute transformation d'un parti qui a ainsi exercé le pouvoir pendant un quart de siècle est impossible:

On ne peut imaginer qu'un parti revient après 25 ans pour faire des choses complètement différentes des choses qu'il faisait avant. Ce n'est pas possible. Ça [ne] se voit pas; ça [ne] se fait pas.

L'Allier cherche ainsi à convaincre l'auditoire que Bertrand ne peut pas transformer véritablement le Progrès civique, qu'il ne peut incarner un «vrai» changement. Par conséquent, Bertrand ne peut briguer la candidature de chef du Progrès civique. Ensuite de façon plutôt abstraite, L'Allier allègue que ce parti est incapable de se transformer. Puis par la technique de l'association, il fait de son adversaire le représentant de la précédente administration du Pro- 
grès civique. Du même coup, Bertrand devient la cible des griefs que l'on peut formuler à l'endroit de son parti.

Pour associer Bertrand au Progrès civique, L'Allier a recours à différents procédés ponctuels. D'abord, il désigne et interpelle fréquemment Bertrand non par son nom mais par son titre: «... le chef du Progrès civique...». Toujours sur un plan formel, L'Allier prétend déceler une filiation entre Bertrand et le Progrès civique: «Vous êtes l'héritier du Progrès civique; vous avez les mêmes virages».

À cet égard, L'Allier épilogue sur le fait que Bertrand pourrait marcher sur les traces de l'ancien conseil municipal. C'est l'affaire de la «Grande Place» qui lui fournit ici ses meilleures munitions. Il fait valoir que Bertrand «a endossé» le projet de l'administration Pelletier:

Monsieur Bertrand a plongé poings et pieds liés dans le projet de la «Grande Place».

Vous avez signé avec Monsieur Pelletier puisque vous avez approuvé sa signature avec enthousiasme. Vous avez dit d'accord.

Vous avez approuvé un projet au sujet duquel vous auriez pu demander de ne pas être coulé dans le ciment tout de suite.

Le type de développement que vous souhaitez poursuivre comme chef du Progrès civique - et je suis content de voir qu'on est plutôt dans la continuité en ce qui vous concerne par rapport à ce qui se faisait auparavant, parce que c'est ça l'enjeu de cette élection: la continuité ou le changement.

À certains moments, de façon explicite, L'Allier déclare Bertrand responsable de décisions prises antérieurement par le Progrès civique:

C'est votre parti qui a creusé le trou [de la «Grande Place»].

Le Progrès civique de Monsieur Bertrand a fait disparaître $d u$ zonage cette obligation de maintenir du logement pour les familles. 
Votre parti ne l'a pas fait dans le passé (financer des maisons de jeunes].

Vous avez dit: «Monsieur Gagnon n'a pas (sic) profité d'aucun avantage de la Ville de Québec et sous mon administration ça va continuer comme ça». Qui peut croire cela?»

Quand L'Allier essaie de peindre un Bertrand solidaire du Progrès civique et responsable de son administration antérieure, il a recours à l'attaque ad hominem dite de «la culpabilité par association» (guilt by association) (Kahane [1988], Johnson et Blair [1983]). Cette forme d'argument se caractérise par le transfert sur l'opposant du discrédit dont fait l'objet un groupe (ou une doctrine) auquel on l'associe.

Presque tous les exemples d'arguments de culpabilité par association qu'étudie la théorie des fallacies relèvent du domaine politique. On y a recours pour que l'adversaire soit jugé en fonction de son entourage, de son groupe de référence ou de son appartenance idéologique. Par exemple, comme le montrent Toulmin, Riecke et Janik [1984], spécifier qu'un opposant est communiste, d'allégeance libérale ou même qu'il est homme ou femme peut, dans certains contextes, constituer une attaque de culpabilité par association, en présupposant que le point de vue d'un communiste, d'un libéral, d'un homme ou d'une femme sur la question traitée est nécessairement biaisé ou erroné. Jason (1988) cite un exemple différent: lors de son débat contre Nixon, Kennedy révèle que le vice-président sortant est appuyé par le Ku Klux Klan, insinuant par là qu'il appartient à l'organisation extrémiste, si bien que l'impopularité relative de ce groupe auprès de la population américaine jette un grave discrédit sur la candidature de Nixon.

La réplique de Bertrand à l'attaque de L'Allier est nettement moins virulente que la réaction de ce dernier à l'offensive du chef du Progrès civique. On peut même constater que, loin de résister vraiment, Bertrand cherche à opérer une sorte de repli stratégique. Au début du débat, il joue manifestement «le changement dans la continuité». Il met ainsi l'accent sur les «... objectifs de renouveau et de changement ...» qu'il dit s'être fixés et avoir atteints: la 
tenue d'un congrès d'orientation, le choix démocratique des candidates et candidats, une augmentation notable du nombre de membres du Progrès civique. Autrement dit, Bertrand affiche une certaine démarcation:

Tout en étant fier de succéder à des hommes de qualité comme Jean Pelletier et Gilles Lamontagne, j'entends bien être de mon temps et adapter notre parti et notre ville à la réalité des années 80 (sic).

Au fur et à mesure du débat, cependant, sous les coups assénés par L'Allier, Bertrand se réclame davantage de la continuité que du changement. Il va ainsi aller jusqu'à appuyer sa candidature sur les réalisations passées du Progrès civique:

... une équipe qui parce qu'elle a livré la marchandise dans le passé va la livrer encore une fois lors des prochaines années.

Dans la même veine, Bertrand va également se porter expressément à la défense de l'administration Pelletier et donc la cautionner, par exemple en matière d'intervention urbaine.

\section{Évaluation de l'argumentation stratégique}

À partir de l'analyse sommaire du débat L'Allier - Bertrand qui précède, je voudrais examiner rapidement quelques aspects de trois différents modes d'évaluation permettant d'apprécier l'argumentation stratégique sur les plans de l'efficacité, de la validité logique et de la moralité.

L'examen des argumentations stratégiques déployées par L'Allier et Bertrand vient corroborer un certain nombre de considérations communément admises au sujet du débat, en particulier quelques traits de la performance des opposants susceptibles d'en infléchir le résultat. Entre autres choses, l'hypothèse selon laquelle une attitude de fermeté et d'attaque serait avantageuse se trouve confirmée. Pour une bonne part, L'Allier est sorti vainqueur du débat parce que, sur le plan de l'argumentation stratégique, il s'est porté avec force à l'offensive plutôt que de s'en tenir à la défensive. 
En particulier, il a réussi à bloquer l'attaque de Bertrand, en contrant la manoeuvre elle-même sans chercher à en réfuter la teneur, et à transformer cette défense en contre-attaque. Dans un débat, il semble bien que, comme au hockey, «la meilleure défense, c'est l'attaque». Pour ne pas voir son argumentation stratégique neutralisée, Bertrand aurait dû l'appuyer davantage en revenant à la charge. Il se contentait, au contraire, de poser une seule fois une affirmation, ne bataillait pas avec L'Allier pour la soutenir et ne cherchait pas non plus de nouvelles façons de l'exploiter.

Si Bertrand a perdu le débat, c'est aussi parce qu'il n'a pas fait preuve d'assez de fermeté ni dans sa défense ni dans son attaque. Il s'est laissé ébranler par l'offensive de l'Allier. Au fur et à mesure du débat, le candidat du «renouveau», au sein même du Progrès civique, s'est transformé, de façon très nette, en apologiste de son action passée. Par là même, Bertrand se trouvait à illustrer précisément la thèse de L'Allier, à savoir qu'il incarnait la continuité plutôt que la rupture avec l'administration sortante. En quelque sorte, l'attitude même de Bertrand a rendu plus crédible l'argumentation stratégique de L'Allier. Dans un débat, une attitude trop défensive et justificatrice donne l'apparence de la faiblesse.

Ce primat de l'attaque sur la défense pourrait expliquer (au moins partiellement) l'intuition suivant laquelle l'aspirant (le challenger) dans un débat jouit au départ d'un avantage. Se trouvant naturellement dans une position offensive, il doit convaincre l'auditoire que le bilan de l'administration sortante est négatif. Sinon, il peut difficilement amener l'électorat à changer de dirigeants. Au contraire, un mandataire qui cherche à se faire réélire est appelé à rendre des comptes; il doit, «défendre son administration». Par la force des choses, son attitude est plus défensive qu'offensive. Cela explique qu'un chef de gouvernement préfère si possible éviter l'affrontement d'un débat. Tel fut le choix de Bourassa en 1989.

Les nouveaux chefs de partis déjà au pouvoir et dont l'administration est critiquable doivent faire valoir un supposé «renouveau» pour ne pas avoir à adopter une attitude de défense ou de justification. Manoeuvre qui est loin d'être facile comme le montre l'exemple de Bertrand au Progrès civique et ceux de PierreMarc Johnson au Parti québécois et John Turner au Parti libéral du 
Canada. Elle est quand même réalisable: Paul Sauvé avec l'Union nationale et Pierre-Elliott Trudeau avec le Parti libéral du Canada l'ont fort bien réussie. Mais contrairement à Bertrand, Johnson et Turner ont résisté au réflexe de justification. À ne considérer que son cas, on peut penser que la plus grave erreur de Bertrand a été de vouloir jouer sur les deux tableaux: profiter du reste de prestige du Progrès civique tout en manifestant une volonté d'en faire une formation politique différente. Le slogan du «renouveau dans la continuité» a joué contre lui: on l'a pris pour quelqu'un qui voulait ménager la chèvre et le chou, attitude peu rentable sur le plan électoral.

Outre son efficacité, la logique d'une argumentation stratégique semble pouvoir être évaluée. Il apparaît légitime de poser la question de sa validité formelle. Dans le cas qui nous occupe, on pourrait essayer de déterminer s'il est logiquement valable de conclure à un manque de crédibilité de la part de L'Allier, du fait qu'il se soit contredit sur un certain nombre de questions. Également peut-on conclure à un manque de crédibilité de la part de Bertrand parce qu'il assume la relève à la direction d'un parti arrivé à la fin d'un long exercice du pouvoir? Il faut, selon toute évidence, répondre négativement à cette question. Sur le plan de la stricte logique, il est tout à fait légitime de changer d'avis comme il est tout aussi légitime de remplacer quelqu'un sans avoir à porter la responsabilité de son mandat. Dans une perspective purement formelle, il faudrait donc rejeter comme étant incorrects les arguments stratégiques déployés par Bertrand et L'Allier.

L'argumentation stratégique ne repose pas sur des arguments logiques. En particulier, les deux attaques ad hominem de mise en contradiction et de culpabilité par association ne sont pas des inférences formelles. Un argument stratégique est un procédé discursif qui se situe hors du cadre logique. C'est ce qui explique probablement que les opposants d'un débat qui se voient confrontés à semblable attaque n'en fassent pas valoir l'illogisme. Maurice Finocchiaro $(1974,1981)$ n'admet pas comme arguments stratégiques les procédés discursifs. Engel (1982) et Walton (1987), prennent la peine de les distinguer des arguments véritablement logiques en les qualifiant d'informal fallacies. 
Par ailleurs, situer les arguments stratégiques hors de la logique n'équivaut pas à leur refuser toute importance dans la vie intellectuelle. Au contraire, comme d'autres modes de pensée quasi logiques (les rapprochements par ressemblance formelle, les associations d'idées et les identifications par proximité géographique) ces arguments se retrouvent dans la plupart des activités intellectuelles, y compris la découverte scientifique, du moins dans sa phase heuristique ${ }^{4}$.

Si les arguments stratégiques échappent à une estimation d'ordre logique, ils semblent par contre pouvoir faire l'objet d'une évaluation d'ordre éthique. C'est le cas des arguments ad hominem, tels ceux que développent L'Allier et Bertrand. Pour toute attaque à la personne se pose la question de savoir si elle est justifiée sur le plan moral: est-ce acceptable éthiquement de s'en prendre non à des idées, mais à celui, à celle ou à ceux qui les défendent? En théorie des fallacies, on subdivise à cette fin, les arguments ad hominem en deux classes: les ad hominem circonstanciels (circumstantial) et les ad hominem abusifs (abusive). Une attaque ad hominem circonstancielle se définit comme une réfutation de l'argument d'un opposant en alléguant que cet argument contredit une autre position, une autre action ou, plus globalement, un autre trait circonstanciel se rapportant à l'opposant. Un argument ad hominem abusif est une tentative visant à disqualifier la position d'un adversaire sans même la discuter et en s'attaquant plutôt directement à la personne de l'opposant. Cette forme d'argumentation est souvent appelée, en anglais, character assassination.

L'argument ad hominem circonstanciel est tout à fait justifié moralement, il n'y a rien de répréhensible à mettre en cause une thèse ou un argument en raison de son incompatibilité avec quelque aspect relatif à l'argumentateur. L'argument ad hominem abusif est d'une légitimité éthique plus douteuse. Cependant, certains auteurs, comme Walton (1987) et Kahane (1988), soutiennent que même des arguments ad hominem abusifs peuvent être, dans certains contextes, moralement légitimes. Kahane, par exem-

4. Je dois cette observation à François Demers. 
ple, prétend qu'il est tout à fait justifié de chercher à discréditer l'argument d'un spécialiste en mettant en cause la compétence qu'on lui attribue dans la matière qui fait l'objet de la discussion. S'il peut être démontré que le spécialiste ne possède pas de véritable compétence, sa position mérite d'être déconsidérée, ou du moins d'être reconsidérée à la baisse.

Les argumentations stratégiques déployées par L'Allier et Bertrand relèvent de l'attaque ad hominem abusive: elles mettent en cause la personne même de l'un et l'autre adversaire dans le but de discréditer leur candidature respective. On ne peut cependant conclure que l'argument de mise en contradiction faite par Bertrand et l'argument de culpabilité par association utilisé par L'Allier sont nécessairement condamnables sur le plan éthique. Au contraire, il semble possible de considérer ces attaques ad hominem comme légitimes sur le plan moral. J'en prends pour preuve le fait que les opposants eux-mêmes, aussi bien que le modérateur et le public, acceptent que de tels arguments soient formulés. Les attaques de mise en contradiction et de culpabilité par association semblent considérées par tous les intervenants comme normales dans un débat électoral et, plus largement, dans l'affrontement politique. Tout se passe comme s'il était de bonne guerre de recourir à ce type d'arguments, sans par ailleurs que soit prise véritablement en compte la question de leur bien-fondé, c'est-à-dire de leur validité logique.

De là, on aurait tort de conclure que toute argumentation stratégique est toujours moralement acceptable. Certains procédés sont manifestement plus douteux ou plus vicieux que les arguments de Bertrand et L'Allier. On pourrait difficilement imaginer un débat électoral dans lequel il serait légitime de porter une accusation grave que l'on sait être fausse (exemple fictif: «Vous avez commis plusieurs vols de fonds publics, quand vous étiez ministre!») ou encore d'user d'hyperboles métaphoriques (autre exemple moins fictif: «Vous avez du sang sur les mains!»).

Pour juger de la moralité ou de l'immoralité des arguments ad hominem, voire de l'ensemble de l'argumentation stratégique, on pourrait poser comme hypothèse que le contexte est la norme. Selon certains faits saillants ou «traits pertinents» d'une situation, 
il serait éthiquement correct ou non de s'attaquer à la personne d'un adversaire afin de contrer son argumentation sans directement s'en prendre à celle-ci. Selon cette hypothèse ${ }^{5}$, à différents contextes correspondraient différentes normes morales, lesquelles traceraient différentes frontières éthiques. Autrement dit, un ensemble spécifique de règles d'ordre éthique ressortiraient au contexte où il s'applique. Par exemple, le principe d'imputabilité concomitant au système démocratique pourrait bien impliquer que, dans le contexte du débat électoral, il soit justifié de demander aux candidats de rendre des comptes. Si la démocratie impose aux politiciens d'assumer leurs actions passées, il devient légitime d'exiger la même chose sur le plan éthique. En vertu de cette seule règle du jeu, on pourrait peut-être démontrer que les attaques ad hominem portées par L'Allier et Bertrand présentent un véritable caractère éthique.

\section{Conclusion}

Au delà du débat Bertrand - L'Allier, en quoi l'analyse de l'argumentation stratégique peut-elle être de quelque utilité pour l'étude de la communication politique? Entre autres choses, elle pourrait peut-être permettre de faire le pont entre les deux «types d'analyse» des médias, plus particulièrement des émissions politiques et, plus spécifiquement encore, des débats politiques à la télévision, que distingue Patrick Champagne (1989, 1988). Selon cet auteur, «l'analyse interne», d'inspiration sémiologique, a jusqu'à maintenant largement dominé «l'analyse externe», relative aux «productions sociales» des messages publics.

De par sa nature, l'argumentation stratégique relève certes de l'analyse interne: elle est constituée d'énoncés qui composent le

5. Elle relève de ce que Richard Johannesen (1978) appelle la «perspective situationnelle» du jugement éthique en communication, suivant laquelle les critères de l'estimation morale sont constitués d'éléments spécifiques de la situation considérée, comme le rôle et la fonction de l'argumentateur, l'urgence du message, les attentes de l'auditoire et son degré de connaissance des techniques utilisées. 
contenu d'un discours. Eu égard à sa finalité, elle tient cependant de l'analyse externe: la fonction de l'argumentation stratégique est de créer des effets émotifs et de donner de la crédibilité à l'argumentateur pour gagner l'adhésion de l'auditoire.

Le texte qu'on vient de lire vise surtout à fournir une caractérisation de l'argumentation stratégique. Mis à part le court développement consacré à son efficacité, il ne s'arrête guère aux effets des arguments stratégiques sur l'auditoire. C'est à cet aspect que pourrait être consacré un prolongement de la présente étude, qui nécessiterait la mise au point d'un instrument de mesure capable d'évaluer les effets d'un débat et des argumentations stratégiques qui y sont développées. 


\section{Bibliographie}

BARTH, E.M., MARTENS, J.L., "Argumentum Ad Hominem: from Chaos to Formal Dialectic», Logique et analyse, 1977, pp. 76-96.

CHAMPAGNE, Patrick, «Qui a gagné? Analyse interne et analyse externe des débats politiques à la télévision», Mots. Les langages $d u$ politique, «La télévision politique», 20 septembre, 1989, pp. 5-21.

CHAMPAGNE, Patrick, «Le cercle politique. Usages sociaux des sondages et nouvel espace politique», Actes de la recherche en sciences sociales, mars, 1988, pp. 71-72.

DEMERS, François, «L'acquisition d'information par le moyen des médias lors d'une campagne électorale, le cas des élections municipales de Québec en novembre 1989», inédit.

ENGEL, Morris, With Good Reason. An Introduction to Informal Fallacies, New York, St Martin's Press, 1982 (1980).

FINOCCHIARO, Maurice, «Fallacies and the Evaluating of Reasoning», American Philosophical Quarterly, 18, 1, 1981, pp. 13-22.

FINOCCHIARO, Maurice, «The Concept of Ad Hominem Argument in Galileo and Locke», Philosophical Forum, 5, 1974, pp. 394-404.

GERBER, D., «On Argumentum Ad Hominem», The Personalist, 55, 1974, pp. 23-29.

GOVIER, Trudy, «Ad Hominem: Revising the Textbooks», Teaching Philosophy, 6, 1, 1983, pp. 13-24.

HAMBLIN, Charles, Fallacies, London, Methuen, 1970.

JASON, Gary, «Are Fallacies Common? A Look at Two Debates», dans Govier, Trudy (Ed.), Selected Issues in Logic and Communication, Belmont, Wadsworth, pp. 20-34.

JENSEN, Vernon, Argumentation. Reasoning in Communication, New York, Van Nostrand, 1981.

JOHANNESEN, Richard, Ethics in Human Communication, Columbus, Ohio, Charles Merril, 1978 (1975). 
JOHNSON, Ralph, Blair, Anthony, Logical Self-Defense, Toronto, McGraw Hill, 1983 (1977).

KAHANE, Howard, Logic and Contemporary Rhetoric. The Use of Reason in Everyday Life, 5e édition, Belmont, Wadsworth, 1988 (1971).

KRAUS, Sydney, DAVIS, Dennis, «Political Debates», dans Nimmo et Sanders, 1981, pp. 273-296.

LAVIGNE, Alain, «Accès aux médias et marketing électoral: le cas du rassemblement populaire de Québec de 1985 et 1989», inédit.

NIMMO, Dan, SANDERS, Keith, Handbook of Political Communication, Beverly Hills, London, Sage, 1981.

PERELMAN, Chaïm, OLBRECHTS-TYTECA, Lucie, Traité de l'argumentation. La nouvelle rhéthorique, Bruxelles, Éditions de l'Université de Bruxelles, 1958.

SPROULE, Michael, Argument. Language and its Influence, New York, McGraw-Hill, 1980.

TOULMIN, Stephen, The Uses of Argument, Cambridge, Cambridge University Press, 1958.

TOULMIN, Stephen, RIECKE, Richard, JANIK, Allan, An Introduction to Reasoning, Macmillan \& Collier, 1978.

van EEMEREN, Frans, GROOTENDORST, Rob, KRUIGER, Tjark, Handbook of Argumentation Theory, Dordrecht, Foris, 1987.

WALTON, Douglas, Informal Fallacies. Towards a Theory of Argument Criticisms, Amsterdam/Philadelphia, John Benjamins, 1987.

WALTON, Douglas, WOODS, John, Argument: the Logic of the Fallacies, Toronto, McGraw-Hill, 1982.

WALTON, Douglas, WOODS, John, «Ad Hominem», The Philosophical Forum, 8, 1977a, pp. 1-20.

WALTON, Douglas, WOODS, John, «Ad Hominem, Contra Gerber», The Personalist, 58, 1977b, pp. 141-6. 